\title{
The history, present situation, and future directions of neoadjuvant chemotherapy for HER2-negative breast cancer
}

\author{
Masahiro Oikawa ${ }^{1,2}$ \\ ${ }^{1}$ The Department of Breast Surgery, New-wa-kai Oikawa Hospital, Fukuoka, Japan; ${ }^{2}$ The Department of Surgical Oncology, Nagasaki University \\ Hospital, Nagasaki, Japan \\ Correspondence to: Masahiro Oikawa. Hirao 2-21-16, Chuou-ku, Fukuoka, 810-0014, Japan. Email: moikawa@oikawahp.or.jp.
}

\begin{abstract}
Chemotherapy has played a significant role in breast cancer therapy and dramatically improved the outcome of breast cancer patients. Neoadjuvant chemotherapy (NAC) is defined as chemotherapy conducted before surgery. The rationale for NAC in operable breast cancer is that the benefit of systemic chemotherapy on the long-term prognosis does not change, regardless of whether chemotherapy is conducted before or after surgery. NAC is now widely used for early and advanced breast cancer patients since it has two significant advantages over conventional adjuvant chemotherapy after surgery. One is that the rate of the breast-conserving surgery increase resulted from tumor shrinkage during NAC, which can contribute to a minimal invasion from surgical therapy and good quality of life following therapy. Another is that a response to chemotherapy can be observed in in-vivo setting. Patients who achieve the pathological complete response (pCR) show a favorable prognosis. Thus, the response to NAC can be used as an indicator for personalized medicine. To date, novel agents for NAC have been explored in randomized trials, setting the pCR rate as an endpoint. Also, response- and residual disease-guided therapy have been ready for prime time in daily practice. The author describes the history, current situation, and future direction of NAC for HER2-negative breast cancer in the review article.
\end{abstract}

Keywords: Breast cancer; neoadjuvant chemotherapy (NAC); pathological complete response (pCR); responseand residual disease-guided therapy

Submitted Jan 09, 2020. Accepted for publication Jun 05, 2020.

doi: $10.21037 /$ cco-20-12

View this article at: http://dx.doi.org/10.21037/cco-20-12

\section{Introduction}

Breast cancer is a systemic disease (Fisher's theory). For completely curing this disease, the combination of systemic therapy to eradicate possible micrometastases with local therapy such as surgery and radiotherapy is crucial. In this context, chemotherapy has dramatically improved the outcomes of breast cancer treatment (1).

Neoadjuvant chemotherapy (NAC) is defined as chemotherapy conducted before surgery. It is now widely used for early- and advanced-stage breast cancer patients (2-4). NAC has two significant advantages over adjuvant chemotherapy. First, the rate of breast-conserving surgery increases as a result of tumor shrinkage during NAC, which can contribute to a minimal invasion from surgical therapy and a good quality of life following therapy. Second, the response to chemotherapy can be observed in in vivo settings, which provides us with valuable information regarding prognosis and response-guided systemic therapy to reduce recurrence.

In this review article, the author describes the history, current situation, and future directions of NAC for HER2negative breast cancer.

\section{The trajectory of NAC for HER2-negative breast cancer}

In the early days of NAC, locally advanced cases were considered as an indication of an operable tumor as a result of down-staging by chemotherapy. Several non- 
randomized studies of NAC showed favorable response rates and safety profiles (5-7). For testing the efficacy and safety of NAC, randomized trials comparing NAC to adjuvant chemotherapy using the same agents as NAC were conducted with regimens that did not include anthracyclines (8-11). In a randomized trial performed by researchers in London (8), 210 breast cancer patients were randomly allocated to the neoadjuvant and post-treatment arms. In both arms, estrogen-negative and estrogen-positive patients were assigned to chemotherapy (MMM: mitozantrone, mitomycin $\mathrm{C}$, and methotrexate) and endocrine therapy (goserelin or formestane), respectively. The differences in disease-free survival (DFS) and overall survival (OS) between the neoadjuvant and post-treatment arms were not significant. Among the estrogen-negative patients who received NAC, the clinical response and complete response (CR) rates were $60 \%$ and $34 \%$, respectively. It is interesting that in the trial designed in the early 1990s, the concept of biology-oriented therapy was already implemented, and the response rate was acceptable even though the regimen did not contain anthracyclines or taxanes, which are currently standard reagents for breast cancer therapy.

After anthracycline-containing regimes became a standard therapy for adjuvant chemotherapy (1), randomized studies comparing NAC to adjuvant chemotherapy containing anthracyclines were tested (12-15). In the pivotal study NSABP B-18 (15), 1,523 patients with operable breast cancer (T1-3 N0-1 M0) were randomly assigned to receive surgery followed by four cycles of AC (doxorubicin and cyclophosphamide) or the same chemotherapy followed by surgery. With a mean observational period of nine and a half years, the OS was $70 \%$ at nine years in the NAC group and $69 \%$ in the adjuvant group $(\mathrm{P}=0.80)$. The DFS was $53 \%$ at nine years in the NAC group and $55 \%$ in the adjuvant group $(\mathrm{P}=0.50)$, which was not statistically significant. The rate of breast conservation surgery was higher in the NAC group (68\%) than in the neoadjuvant group $(60 \%, \mathrm{P}=0.001)(16)$. However, there was a trend toward a higher rate of ipsilateral breast recurrence in the NAC group compared to the neoadjuvant group $(10.7 \%$ and $7.6 \%$, respectively, $\mathrm{P}=0.12$ ). This finding was confirmed by another large scale randomized study, EORTC10902 (14). In the study, 698 patients (T1c-4b N0-1 M0) were randomly assigned to four cycles of FEC (fluorouracil, epirubicin, and cyclophosphamide) preoperatively or after surgery. After a mean observation period of 56 months, the OS and DFS at four years in the NAC group and the adjuvant group were $82 \%$ and $84 \%(\mathrm{P}=0.38)$ and $65 \%$ and $70 \%(\mathrm{P}=0.27)$, respectively. Contrary to the NSBABP B-18 trial, the local recurrence rate did not differ between groups in this study $(\mathrm{P}=0.61)$. Importantly, both studies showed that patients achieved a clinically CR in the NAC group, which showed a significantly better prognosis in OS and DFS; thus, the pathological complete response (pCR) was considered a surrogate endpoint of prognosis in the NAC studies.

These large-scale randomized studies enabled researchers of the Early Breast Cancer Trialists Collaborative Group (EBCTCG) to exam a patient-based meta-analysis comparing NAC to adjuvant chemotherapy (17). In a metaanalysis, the OS and DFS of 4756 women in 10 randomized trials of operable breast cancer were compared between NAC and the same chemotherapy given postoperatively. The results provided two compelling points of evidence for NAC: firstly, the OS and DFS did not change regardless of whether the chemotherapy was given before or after surgery. Secondly, the rate of breast-conserving surgery was higher in the NAC group, although the locoregional recurrence was higher in the NAC group. Based on the evidence supporting the safety of NAC, it was considered standard therapy and not experimental. Currently, candidates for NAC are patients who should receive adjuvant chemotherapy, particularly those who want to have breast-conserving surgery. Thus, almost all patients with triple-negative breast cancer except for tiny invasive areas (e.g., less than $5 \mathrm{~mm}$ ) are suitable candidates for NAC.

After the safety of NAC was confirmed, determining which regimen should be used in NAC have become areas of high interest. Given the evidence that the addition of taxanes to anthracyclines in adjuvant chemotherapy has improved outcomes (18), several randomized trials to test the efficacy of taxanes in the NAC setting were designed. In an Italian study (19), 1,355 patients with breast cancer (T2-3 N0-1 M0) were randomly assigned to three treatment arms: arm A: four cycles of doxorubicin and CMF were sequentially administered, followed by surgery; arm B: four cycles of AT (doxorubicin and paclitaxel) and CMF were sequentially administered, followed by surgery; and arm C: four cycles of AT and CMF were sequentially administered before surgery. The results showed that the addition of paclitaxel to adjuvant doxorubicin followed by CMF significantly improved relapse-free survival (RFS) and distant RFS but not OS (arm A vs. arm B). There was no difference in RFS, distant RFS, or OS between NAC and adjuvant chemotherapy (arm B vs. arm C). However, the rate of breast-conserving surgery was significantly higher in the NAC group. In the Aberdeen study (20), 162 patients 
with operable breast cancer (T2-4 N0-2 M0) received four cycles of CVAP (cyclophosphamide, vincristine, doxorubicin, and prednisolone) as NAC. Then, 104 patients who achieved a clinical CR or PR randomly received four cycles of the same CVAP or docetaxel before surgery. The overall response rate (ORR) and the pCR rate in the CVAP and docetaxel groups were $64 \%$ and $85 \%$ and $15.4 \%$ and $30.8 \%$, respectively. The patients receiving docetaxel had an increased breast conservation rate $(67 \% v s .48 \%)$ and an increased survival at a median follow-up of three years (21). Meanwhile, the other patients who did not display a response to the CVAP (clinical SD or PD) received four cycles of docetaxel before surgery. The ORR and pCR were $55 \%$ and $2 \%$, which implied the potential benefit of response-guided therapy.

The NSABP B-27 trial examined the efficacy of the addition of docetaxel to AC-based NAC (22). The 2411 patients with operable breast cancer (T1c-3 N0-1 M0 or T1-3 N1 M0) were randomly assigned to receive four cycles of AC before surgery (group 1), AC followed by $\mathrm{T}$ (docetaxel) and surgery (group 2), or AC followed by surgery and $\mathrm{T}$ after surgery (group 3 ). After a median follow-up of 77.9 months, there were no statistically significant differences in prognosis according to treatment. The five-year DFS in groups 2 and 3 was better than that in group 1, although the difference was not significant. The rate of pCR was higher in group 2 than in groups 1 and $3(26.1 \%, 12.9 \%$, and $14.4 \%$, respectively). Thus, the addition of docetaxel to NAC improved the pCR rate. Although the addition of docetaxel to NAC did not improve the OS and DFS despite doubling the pCR rate, patients achieving pCR showed significantly better OS and DFS (HR 0.33 and 0.45 , respectively).

Based on the evidence from the randomized studies mentioned above, the administration of taxane, in addition to anthracycline, increases the pCR rate. Given the high correlation between the pCR rate and better OS and DFS, the sequential use of anthracyclines and taxane is the standard regimen for NAC. Furthermore, the pCR rate has become regarded as the surrogate marker of survival in NAC studies to investigate novel agents added to the standard regimen.

\section{The exploration of novel agents for NAC}

Entering the 21st century, several novel agents for NAC were explored in randomized trials with the pCR rate considered the endpoint. In the GeparQuattro trial
$(23,24), 1,421$ patients with breast cancer who should be candidates for adjuvant chemotherapy (e.g., cT3-4 Nany M0 or ER- and/or PgR-negative or cT2 cN1 M0 with ER- and/or PgR-positive, or cT1 pN1 M0 with ER- and/or PgR-positive) received four cycles of EC and were then randomized to either four cycles of docetaxel (EC-T), four cycles of docetaxel and capecitabine (ECTX), or four cycles of docetaxel followed by capecitabine (EC-T-X) before surgery. Approximately one-third of patients had HER2-positive breast cancer and received trastuzumab concomitantly with all cycles. The results showed that there was no significant difference in the pCR rate $(22.3 \%, 19.5 \%$, and $22.3 \%$, respectively). The rate of breast-conserving surgery was similar as well $(67.8 \%$, $66.9 \%$, and $63.5 \%$, respectively). Outcomes did not improve for patients in the EC-TX group (DFS: HR 0.92, $\mathrm{P}=0.463$; OS: HR 0.93, $\mathrm{P}=0.618$ ) or for patients in the EC-T-X group (DFS: HR 0.97, $\mathrm{P}=0.818$; OS: HR 0.97, $\mathrm{P}=0.825)$. In the OOTR N003 trial (25), 477 patients with operable breast cancer (T1c-3 N0-1 M0) were randomly assigned to receive four cycles of docetaxel with or without capecitabine following four cycles of FEC (fluorouracil, epirubicin, and cyclophosphamide) before surgery. There were no significant differences in the pCR rate (docetaxel/ capecitabine: $23 \%$; docetaxel: $24 \%$; $\mathrm{P}=0.748)$, DFS, or OS. The rate of breast-conserving surgery was almost identical in the two groups (70.7\% vs. $71.4 \%)$.

In the GeparQuinto trial $(26,27)$, which had the same eligibility criteria as the GeparQuattro trial, 1948 patients with HER2-negative breast cancer were randomly assigned to receive four cycles of EC followed by four cycles of docetaxel with or without the concomitant use of bevacizumab. The pCR rate, the primary endpoint, was significantly higher in the bevacizumab group than in the control group ( $18.4 \%$ vs. $14.9 \%, \mathrm{P}=0.04)$. The difference in the pCR rate was particularly noticeable in the triplenegative breast cancer patients $(39.3 \%$ vs. $27.9 \%, \mathrm{P}=0.003)$. However, with a median follow-up of 3.8 years, the threeyear DFS and OS rates did not differ for patients receiving bevacizumab compared with the control group (DFS: HR 1.03, $\mathrm{P}=0.784$; OS: HR 0.974, $\mathrm{P}=0.842)$.

In the NSABP B-40 trial $(28,29), 1206$ patients with operable breast cancer (T1c-3 N0-2a M0) were randomly assigned to receive four cycles of docetaxel (T-AC), docetaxel plus capecitabine (TX-AC), or docetaxel plus gemcitabine (TG-AC) followed by four cycles of AC with or without bevacizumab before surgery. The pCR rate in the patients with bevacizumab was significantly higher than 
that in the patients without bevacizumab (34.5\% vs. $28.2 \%$, $\mathrm{P}=0.02)$. The $\mathrm{pCR}$ rates for the T-AC, TX-AC, and TG-AC groups were $32.7 \%, 29.7 \%$, and $31.8 \%$, respectively, which did not differ significantly. After a median follow-up of 4.7 years, neither capecitabine nor gemcitabine, in addition to neoadjuvant T-AC, increased the DFS or OS. The addition of bevacizumab significantly increased OS $(\mathrm{P}=0.004)$ but did not significantly increase DFS $(\mathrm{P}=0.06)$. However, a metaanalysis to examine the efficacy of neoadjuvant bevacizumab plus chemotherapy $(\mathrm{BEV}+\mathrm{CT}) v s$. chemotherapy $(\mathrm{CT})$ alone for the treatment of HER2-negative breast cancer, including two previous randomized studies, showed that the pCR rate and five-year DFS were higher for the BEV + CT group $(\mathrm{P}=0.001$ and 0.020 , respectively); however, the fiveyear OS rate showed no significant difference $(\mathrm{P}=0.18)(30)$.

After the efficacy of platinum agents for metastatic triple-negative breast cancer was reported (31), platinum agents were tested in the NAC setting. Several nonrandomized trials with NAC regimens containing platinum salts showed a reasonable pCR rate with triple-negative breast cancer (32-34). Notably, the patients with BRCA1 or BRCA2 mutations showed a high pCR rate, likewise clinical trial in the metastatic setting. In the CALGB 40603 (Alliance) study (35), which was a $2 \times 2$ factorial, open-label, randomized phase II trial, the impact of adding carboplatin and/or bevacizumab was evaluated. A total of 443 patients with operable triple-negative breast cancer (Stage II to III) were randomly assigned to receive 12 cycles of weekly paclitaxel with or without bevacizumab or 12 cycles of weekly paclitaxel and 4 cycles of carboplatin with or without bevacizumab. The primary endpoint was the pCR rate, which was significantly higher in either the carboplatin (60\% vs. $44 \% ; \mathrm{P}=0.0018)$ or bevacizumab group $(59 \%$ vs. $48 \% ; \mathrm{P}=0.0089$ ). However, the long-term outcomes of this study were not reported. In the GeparSixto trial $(36,37)$, 315 patients with operable triple-negative breast cancer (Stage II to III) were randomly assigned to receive 18 cycles of weekly paclitaxel, non-pegylated liposomal doxorubicin, and six cycles of bevacizumab with or without 18 cycles of carboplatin followed by surgery. The pCR rate in the group with carboplatin was significantly higher than that in the group without carboplatin $(53.2 \%$ vs. $36.9 \%, \mathrm{P}=0.005)$. In the secondary analysis of the study $(\mathrm{n}=291)$, the pCR rate was higher in the patients with BRCA1 or BRCA2 mutations than in the patients without these mutations (66.7\% vs. $36.4 \%, \mathrm{P}=0.008$ ). However, improvement in the pCR rate with the addition of carboplatin was observed only in the patients without BRCA1 or BRCA2 mutations.
After a median follow-up of 35 months, a preferable DFS rate was observed in the carboplatin group compared with the non-carboplatin group ( $\mathrm{HR}=0.55, \mathrm{P}=0.03$ ). Similar to the pCR rate, the improvement in the DFS rate with the addition of carboplatin was observed only in the patients without BRCA1 or BRCA2 mutations.

For other agents for triple-negative breast cancer, poly ADP-ribose polymerase (PARP) inhibitor was effective in metastatic breast cancer patients with BRCA1 or BRCA2 mutations (38). In the I-SPY 2 trial (39), 72 patients with breast cancer were randomly assigned to receive 12 cycles of weekly paclitaxel or weekly paclitaxel plus carboplatin and veliparib followed by four cycles of AC and surgery. The pCR rate in the triple-negative patients was $51 \%$ and $26 \%$, respectively. Conversely, the patients with HER2-negative and hormone receptor-positive breast cancer did not benefit from veliparib and carboplatin. A phase III trial is planned to confirm the benefits of veliparib in the NAC setting with a focus on patients with operable triple-negative breast cancer.

Immuno-checkpoint inhibitors show efficacy for the socalled hot tumor [e.g., high tumor mutation burden, high tumor-infiltrating lymphocyte (TILs)]. For metastatic triple-negative breast cancer, the immuno-checkpoint inhibitor atezolizumab improved progression-free survival in combination with nab-paclitaxel (40). In the GeparNuevo study (41), 174 patients with operable triple-negative breast cancer (cT1b-cT4a-d) were randomly assigned to receive 12 cycles of weekly nab-paclitaxel followed by 4 cycles of dose-dense EC with or without concomitant durvalumab every four weeks before surgery. At first, the trial planned a window phase, in which durvalumab or placebo monotherapy was given two weeks before the start of chemotherapy. However, the independent data monitoring committee recommended amending the study design, and the window phase was stopped after 117 patients were recruited. In total, the pCR rate was $53.4 \%$ and $44.2 \%$, and the difference was not significant $(\mathrm{P}=0.287)$. When focusing on the patients with the window phase, the pCR rate in the durvalumab group was significantly higher than that in the control group $(61.0 \%$ vs. $41.4 \%, \mathrm{P}=0.035)$. In the KEYNOTE-522 study, 1,174 patients with operable triple-negative breast cancer (T1c N1-2 or T2-4 N0-2) were randomized 2:1 to the experimental and control arms to receive four cycles of paclitaxel and carboplatin followed by EC or AC with or without concomitant pembrolizumab prior to surgery. After a median follow-up of 15.5 months, the pCR rate with pembrolizumab and placebo was $64.8 \%$ 
and $51.2 \%$, respectively. The difference was statistically significant irrespective of the PDL-1 expression. The event-free survival rate showed a preferable trend in the pembrolizumab group compared with the control arm (42).

In summary, no cytotoxic agents, except for carboplatin for triple-negative breast cancer, provided a benefit in addition to the sequential use of anthracyclines and taxane in the NAC setting. The addition of bevacizumab to NAC improved the pCR rate but has not translated to an improvement in OS. Platinum salts may play an essential role in the subset population of triple-negative breast cancer. However, whether the germ-line mutations at BRCA1 or BRCA2 are predictive markers remains unclear. The roles of the PARP inhibitor and immuno-checkpoint inhibitors are promising, particularly for triple-negative breast cancer. The results from randomized phase III studies are expected before the implementation of these agents to daily practice.

\section{PCR rate as a predictive marker}

As mentioned above, the pCR rate is regarded as an endpoint representing long-term outcomes in clinical studies to identify new drugs for NAC. However, metaanalyses of NAC studies that use pCR as an endpoint have shown that the pCR rate differs significantly between the subtypes (43). Therefore, the meaning of the pCR rate differs depending on the subtypes. In a meta-analysis by the GBG and AGO-B, the pCR rate was associated with improved DFS in ER-positive of grade 2 or 3 and HER2negative tumors $(\mathrm{P}=0.005)$, ER-negative and HER2positive tumors $(\mathrm{P}=0.001)$, and triple-negative tumors $(\mathrm{P}=0.001)$ but not in ER-positive of grade 1 and HER2negative $(\mathrm{P}=0.39)$ or ER-positive and HER2-positive $(\mathrm{P}=0.45)$ breast cancer (44). In a meta-analysis consisting of 12 randomized trials including 11,955 patients, the pCR rate was associated with long-term outcomes (OS, DFS). The association between the pCR rate and longterm outcomes was most influential in patients with triplenegative breast cancer and ER-negative and HER2-positive breast cancer; conversely, it was not significant in patients with ER-positive of grade 1 or 2 and HER2-negative breast cancer. Although improvement in the pCR rate predicts superior long-term outcomes in patient-level analyses, this did not translate to beneficial long-term outcomes in a triallevel analysis (45). Based on the results from meta-analyses, setting the pCR rate, which is postulated to be a surrogate marker of long-term outcomes, as a primary endpoint in a NAC study can be justified when the cancer type is aggressive (e.g., triple-negative and HER2-enrich) as long as the long-term outcomes will be assessed.

For patients with ER-positive and HER2-negative breast cancer, the necessity of NAC depends on the risk of recurrence (ROR), similar to adjuvant chemotherapy. In the adjuvant setting, multi-gene assays successfully segregate high-risk patients who can receive a survival benefit by chemotherapy (46-49). These gene-expression analyses have been tested in the NAC setting as well. In a prospective non-randomized study, 97 patients with ER-positive and HER2-negative breast cancer received taxane-based NAC, and gene expression from core-needle biopsies before the initiation of the therapy was tested for the prediction of the response. Clinical CR was significantly related to a high recurrence score $(\mathrm{P}=0.008)$ (50). Another study in which 60 patients with ER-positive and HER2-negative breast cancer received anthracycline/ taxane-based NAC showed no statistically significant association with the clinical response when assessed as a recurrence score classified as a categorical or continuous variable $(\mathrm{P}=0.21)(51)$.

In a study examining the predictive ability of MammaPrint for the response to NAC (52), among 167 patients, $144(86 \%)$ had a poor prognosis signature and $23(14 \%)$ had a good prognosis signature. None of the patients with a good prognosis signature $(n=23)$ achieved a pCR, whereas 29 patients $(20 \%)$ in the poor prognosis signature group $(\mathrm{n}=144)$ did $(\mathrm{P}=0.015)$.

In another study conducted by Parker et al., expressionbased intrinsic subtypes and the ROR score from 133 core-needle biopsies before the initiation of NAC were compared to assess the pCR rate (53). The results showed that the ROR score achieved a $94 \%$ sensitivity and $97 \%$ negative predictive value for identifying non-responders to chemotherapy.

In another study, Tsunashima et al. constructed an expression-based prediction model for the pCR rate to NAC with the expression data from 363 core-needle biopsies obtained before NAC from their own or public dataset (54). They developed a prediction model for pCR named MPCP155, which included 155 genes; this model was found to have an $88 \%$ sensitivity and $97.4 \%$ negative predictive value in the validation dataset.

Multigene assays have the potential to segregate patients who have a chemo-sensitivity in both the adjuvant and the NAC setting. In the near future, multigene assay-guided NAC (55) will be implemented in daily practice after prospective randomized studies. 


\section{Response- and residual disease-guided therapy for NAC}

One of the benefits of NAC is that the response to chemotherapy can be observed in in vivo settings, which has not been conducted in daily practice yet. However, several randomized studies provided us the survival advantages of response-guided therapy after NAC.

In the GeparTrio trial (56), 2,072 patients with operable or local advanced breast cancer received two cycles of TAC (docetaxel, doxorubicin, and cyclophosphamide). Then, early responders were randomly assigned to receive four or six cycles of additional TAC, whereas non-responders were randomly assigned to receive four cycles of NX (vinorelbine and capecitabine) or four cycles of additional TAC. Among the responders, the additional six cycles of TAC did not improve the pCR rate ( $24 \%$ vs. $21 \%$ ). However, DFS significantly improved in the TAC group receiving six cycles. Likewise, among the non-responders, changing to the NX regime did not improve the pCR rate (6\% vs. 5\%), whereas DFS significantly improved in the NX group.

Similar to the response-guided therapy, residual diseaseguided therapy, defined as additional adjuvant therapy for patients with residual cancer cells after NAC, is also promising. In the CREATE-X trial (57), 910 patients with HER2-negative breast cancer who did not achieve pCR after NAC (containing anthracycline, taxane, or both) were randomly assigned to receive standard postsurgical treatment with or without capecitabine. In total, the addition of capecitabine after surgery improved the DFS and OS (HR 0.70 and 0.59 , respectively). In particular, patients with triple-negative breast cancer showed significant improvement in DFS and OS (HR 0.58 and 0.52, respectively).

In the POTENT trial (58), 1939 patients with ERpositive and HER2-negative breast cancer with an intermediate or high ROR, which includes the patients who did not achieve pCR after NAC, were enrolled. Patients were randomly assigned to receive standard adjuvant endocrine therapy with or without a one-year administration of S-1.

After a median follow-up of 51.4 months, S-1 significantly reduced invasive events (HR 0.63, $\mathrm{P}=0.003$ ). The estimated five-year invasive DFS rates in the S-1 and control groups were $86.9 \%$ and $81.5 \%$, respectively.

The results of these trials clearly demonstrate the efficacy of response- and residual disease-guided therapy. Therefore, it should be implemented in daily practice during or after NAC.

\section{Conclusions}

With plenty of evidence from randomized studies on NAC, the safety and advantages of NAC have been confirmed. Almost all patients with triple-negative operable breast cancer should be candidates for NAC with anthracycline and taxane. To improve the pCR rate, new drugs will be integrated into the NAC regimen. Among them, immunocheckpoint inhibitors for so-called "hot tumors" and PARP inhibitors for BRCA1/2 mutation-positive tumors are promising in the future. For patients with operable ERpositive and HER2-negative breast cancer, the indication for NAC is the same as that for adjuvant chemotherapy. Risk assessment using multi-gene assays can stratify the patients with high-risk and chemo-sensitive tumors who are candidates for NAC. Conversely, patients with high-risk but non-chemo-sensitive tumors may be good candidates for neoadjuvant hormonal therapy with or without targeted therapy, such as CDK 4/6 inhibitors.

Response- and residual disease-guided therapy is ready to play a key role in NAC treatment. For patients who do not achieve pCR after a standard regimen of NAC with triplenegative and ER-positive and HER2-negative breast cancer, additional adjuvant capecitabine and hormonal therapy with S-1, respectively, can improve long-term outcomes. New chemo-regimens with response- and residual diseaseguided therapy will be examined in randomized studies and implemented into daily practice in the future.

\section{Acknowledgments}

Funding: This work was supported by a JSPS KAKENHI Grant-in-Aid for Scientific Research (C) (19K090751).

\section{Footnote}

Provenance and Peer Review: This article was commissioned by the Guest Editors (Yutaka Yamamoto and Takayuki Ueno) for the series "Neoadjuvant/Adjuvant Treatment for Early Breast Cancer" published in Chinese Clinical Oncology. The article was sent for external peer review organized by the Guest Editors and the editorial office.

Conflicts of Interest: The author has completed the ICMJE uniform disclosure form (available at http://dx. doi. org/10. 21037/(cco-20-12). The series "Neoadjuvant/ 
Adjuvant Treatment for Early Breast Cancer" was commissioned by the editorial office without any funding or sponsorship. The author has no other conflicts of interest to declare.

Ethical statement: The author is accountable for all aspects of the work in ensuring that questions related to the accuracy or integrity of any part of the work are appropriately investigated and resolved.

Open Access Statement: This is an Open Access article distributed in accordance with the Creative Commons Attribution-NonCommercial-NoDerivs 4.0 International License (CC BY-NC-ND 4.0), which permits the noncommercial replication and distribution of the article with the strict proviso that no changes or edits are made and the original work is properly cited (including links to both the formal publication through the relevant DOI and the license). See: https://creativecommons.org/licenses/by-nc-nd/4.0/.

\section{References}

1. Early Breast Cancer Trialists' Collaborative Group. Polychemotherapy for early breast cancer: an overview of the randomised trials. Lancet 1998;352:930-42.

2. Clough KB, Acosta-Marı'n V, Nos C, et al. Rates of neoadjuvant chemotherapy and oncoplastic surgery for breast cancer surgery: a French national survey. Ann Surg Oncol 2015;22:3504-11.

3. Mougalian SS, Soulos PR, Killelea BK, et al. Use of neoadjuvant chemotherapy for patients with stage I to III breast cancer in the United States. Cancer 2015;121:2544-52.

4. Vugts G, Maaskant-Braat AJ, Nieuwenhuijzen GA, et al. Patterns of care in the administration of neo-adjuvant chemotherapy for breast cancer. a population-based study. Breast J 2016;22:316-21.

5. Bonadonna G, Veronesi U, Brambilla C, et al. Primary chemotherapy to avoid mastectomy in tumors with diameters of three centimeters or more. J Natl Cancer Inst 1990;82:1539-45.

6. De Lena M, Zucali R, Viganotti G, et al. Combined chemotherapy-radiotherapy approach in locally advanced (T3b-T4) breast cancer. Cancer Chemother Pharmacol 1978;1:53-9.

7. Deo SV, Bhutani M, Shukla NK, et al. Randomized trial comparing neo-adjuvant versus adjuvant chemotherapy in operable locally advanced breast cancer (T4b N0-2 M0). J
Surg Oncol 2003;84:192-7.

8. Gazet JC, Ford HT, Gray R, et al. Estrogen-receptordirected neoadjuvant therapy for breast cancer: results of a randomised trial using formestane and methotrexate, mitozantrone and mitomycin C (MMM) chemotherapy. Ann Oncol 2001;12:685-91.

9. Powles TJ, Hickish TF, Makris A, et al. Randomized trial of chemoendocrine therapy started before or after surgery for treatment of primary breast cancer. J Clin Oncol 1995;13:547-52.

10. Taucher S, Steger GG, Jakesz R, et al. The potential risk of neoadjuvant chemotherapy in breast cancer patients-results from a prospective randomized trial of the Austrian Breast and Colorectal Cancer Study Group (ABCSG-07). Breast Cancer Res Treat 2008;112:309-16.

11. Perloff M, Lesnick GJ. Chemotherapy before and after mastectomy in stage III breast cancer. Arch Surg 1982;117:879-81.

12. Mauriac L, MacGrogan G, Avril A, et al. Neoadjuvant chemotherapy for operable breast carcinoma larger than $3 \mathrm{~cm}$ : a unicentre randomized trial with a 124-month median follow-up. Institut Bergonie Bordeaux Groupe Sein (IBBGS). Ann Oncol 1999;10:47-52.

13. Scholl SM, Asselain B, Palangie T, et al. Neoadjuvant chemotherapy in operable breast cancer. Eur J Cancer 1991;27:1668-71.

14. van der Hage JA, van de Velde CJ, Julien JP, et al. Preoperative chemotherapy in primary operable breast cancer: results from the European Organization for Research and Treatment of Cancer trial 10902. J Clin Oncol 2001;19:4224-37.

15. Wolmark N, Wang J, Mamounas E, et al. Preoperative chemotherapy in patients with operable breast cancer: nine-year results from National Surgical Adjuvant Breast and Bowel Project B-18. J Natl Cancer Inst Monogr 2001;(30):96-102.

16. Fisher B, Bryant J, Wolmarket N, et al. Effect of preoperative chemotherapy on the outcome of women with operable breast cancer. J Clin Oncol 1998;16:2672-85.

17. Early Breast Cancer Trialists' Collaborative Group. Long-term outcomes for neoadjuvant versus adjuvant chemotherapy in early breast cancer: meta-analysis of individual patient data from ten randomised trials. Lancet Oncol 2018;19:27-39.

18. Early Breast Cancer Trialists' Collaborative Group (EBCTCG), Peto R, Davies C, et al. Comparisons between different polychemotherapy regimens for early 
breast cancer: meta-analyses of long-term outcome among 100,000 women in 123 randomised trials. Lancet 2012;379:432-44.

19. Gianni L, Baselga J, Eiermann W, et al. Phase III trial evaluating the addition of paclitaxel to doxorubicin followed by cyclophosphamide, methotrexate, and fluorouracil, as adjuvant or primary systemic therapy: European Cooperative Trial in Operable Breast Cancer. J Clin Oncol 2009;27:2474-81.

20. Smith IC, Heys SD, Hutcheon AW, et al. Neoadjuvant chemotherapy in breast cancer: significantly enhanced response with docetaxel. J Clin Oncol 2002;20:1456-66.

21. Heys SD, Hutcheon AW, Sarkar TK, et al. Neoadjuvant docetaxel in breast cancer: 3-year survival results from the Aberdeen trial. Clin Breast Cancer 2002;3:S69-74.

22. Bear HD, Anderson S, Smith RE, et al. Sequential preoperative or postoperative docetaxel added to preoperative doxorubicin plus cyclophosphamide for operable breast cancer: national surgical adjuvant breast and bowel project protocol B-27. J Clin Oncol 2006;24:2019-27.

23. von Minckwitz G, Rezai M, Loibl S, et al. Capecitabine in addition to anthracycline- and taxane-based neoadjuvant treatment in patients with primary breast cancer: phase III GeparQuattro study. J Clin Oncol 2010;28:2015-23.

24. von Minckwitz G, Rezai M, Fasching PA, et al. Survival after adding capecitabine and trastuzumab to neoadjuvant anthracycline-taxane-based chemotherapy for primary breast cancer (GBG 40--GeparQuattro). Ann Oncol 2014;25:81-9.

25. Ohno S, Chow LWC, Sato N, et al. Randomized trial of preoperative docetaxel with or without capecitabine after 4 cycles of 5-fluorouracil- epirubicin-cyclophosphamide (FEC) in early-stage breast cancer: exploratory analyses identify Ki67 as a predictive biomarker for response to neoadjuvant chemotherapy. Breast Cancer Res Treat 2013;142:69-80.

26. von Minckwitz G, Loibl S, Untch M, et al. Survival after neoadjuvant chemotherapy with or without bevacizumab or everolimus for HER2-negative primary breast cancer (GBG 44-GeparQuinto)dagger. Ann Oncol 2014;25:2363-72.

27. von Minckwitz G, Eidtmann H, Rezai M, et al. Neoadjuvant chemotherapy and bevacizumab for HER2negative breast cancer. N Engl J Med 2012;366:299-309.

28. Bear HD, Tang G, Rastogi P, et al. Bevacizumab added to neoadjuvant chemotherapy for breast cancer. N Engl J Med 2012;366:310-20.
29. Bear HD, Tang G, Rastogi P, et al. Neoadjuvant plus adjuvant bevacizumab in early breast cancer (NSABP B-40 [NRG Oncology]): secondary outcomes of a phase 3, randomised controlled trial. Lancet Oncol 2015;16:1037-48.

30. Wei J, Luo Y, Fu D. Early and late outcomes of bevacizumab plus chemotherapy versus chemotherapy alone as a neoadjuvant treatment in HER2-negative nonmetastatic breast cancer: a meta-analysis of randomized controlled trials. Onco Targets Ther 2018;11:9049-59.

31. Tutt A, Tovey H, Cheang MCU, et al. Carboplatin in BRCA1/2-mutated and triple-negative breast cancer BRCAness subgroups: the TNT Trial. Nat Med 2018;24:628-37.

32. Sikov WM, Dizon DS, Strenger R, et al. Frequent pathologic complete responses in aggressive stages II to III breast cancers with every-4-week carboplatin and weekly paclitaxel with or without trastuzumab: a Brown University Oncology Group Study. J Clin Oncol 2009;27:4693-700.

33. Byrski T, Dent R, Blecharz P, et al. Results of a phase II open-label, non-randomized trial of cisplatin chemotherapy in patients with BRCA1-positive metastatic breast cancer. Breast Cancer Res 2012;14:R110.

34. Silver DP, Richardson AL, Eklund AC, et al. Efficacy of neoadjuvant Cisplatin in triple-negative breast cancer. J Clin Oncol 2010;28:1145-53.

35. Sikov WM, Berry DA, Perou CM, et al. Impact of the addition of carboplatin and/or bevacizumab to neoadjuvant once-per-week paclitaxel followed by dosedense doxorubicin and cyclophosphamide on pathologic complete response rates in stage II to III triple-negative breast cancer: CALGB 40603 (Alliance). J Clin Oncol 2015;33:13-21.

36. von Minckwitz G, Schneeweiss A, Loibl S, et al. Neoadjuvant carboplatin in patients with triple-negative and HER2-positive early breast cancer (GeparSixto; GBG 66): a randomised phase 2 trial. Lancet Oncol 2014;15:747-56.

37. Hahnen E, Lederer B, Hauke J, et al. Germline mutation status, pathological complete response, and disease-free survival in triple-negative breast cancer: secondary analysis of the geparsixto randomized clinical trial. JAMA Oncol 2017;3:1378-85.

38. Robson ME, Tung N, Conte P, et al. OlympiAD final overall survival and tolerability results: Olaparib versus chemotherapy treatment of physician's choice in patients with a germline BRCA mutation and HER2-negative metastatic breast cancer. Ann Oncol 2019;30:558-66. 
39. Rugo HS, Olopade OI, DeMichele A, et al. Adaptive randomization of veliparib-carboplatin treatment in breast cancer. N Engl J Med 2016;375:23-34.

40. Schmid P, Adams S, Rugo HS, et al. Atezolizumab and nab-paclitaxel in advanced triple-negative breast cancer. $\mathrm{N}$ Engl J Med 2018;379:2108-21.

41. Loibl S, Untch M, Burchardi N, et al. A randomised phase II study investigating durvalumab in addition to an anthracycline taxane-based neoadjuvant therapy in early triple-negative breast cancer: clinical results and biomarker analysis of GeparNuevo study. Ann Oncol 2019;30:1279-88

42. Schmid P, Cortés J, Dent R, et al. Keynote-522: phase 3 study of pembrolizumab (PEMBRO) + chemotherapy (CHEMO) vs placebo (PBO) + chemo as neoadjuvant treatment, followed by $\mathrm{PEMBRO}$ vs $\mathrm{PBO}$ as adjuvant treatment for early triple-negative breast cancer (TNBC). Ann Oncol 2019;30:v851-934.

43. HOUSSAMI N, Macaskill P, von Minckwitz G, et al. Meta-analysis of the association of breast cancer subtype and pathologic complete response to neoadjuvant chemotherapy. Eur J Cancer 2012;48:3342-54.

44. von Minckwitz G, Untch M, Blohmer JU, et al. Definition and impact of pathologic complete response on prognosis after neoadjuvant chemotherapy in various intrinsic breast cancer subtypes. J Clin Oncol 2012;30:1796-804.

45. Cortazar P, Zhang L, Untch M, et al. Pathological complete response and long-term clinical benefit in breast cancer: the CTNeoBC pooled analysis. Lancet 2014;384:164-72.

46. Sparano JA, Gray RJ, Makower DF, et al. Adjuvant chemotherapy guided by a 21-gene expression assay in breast cancer. N Engl J Med 2018;379:111-21.

47. van de Vijver MJ, He YD, van 't Veer LJ, et al. A geneexpression signature as a predictor of survival in breast cancer. N Engl J Med 2002;347:1999-2009.

48. Dowsett M, Sestak I, Lopez-Knowles E, et al. Comparison of PAM50 risk of recurrence score with oncotype DX and IHC4 for predicting risk of distant recurrence after endocrine therapy. J Clin Oncol 2013;31:2783-90.

49. Naoi Y, Kishi K, Tanei T, et al. Development of 95-gene

Cite this article as: Oikawa M. The history, present situation, and future directions of neoadjuvant chemotherapy for HER2negative breast cancer. Chin Clin Oncol 2020;9(3):29. doi: $10.21037 /$ cco-20-12 classifier as a powerful predictor of recurrences in nodenegative and ER-positive breast cancer patients. Breast Cancer Res Treat 2011;128:633-41.

50. Chang JC, Makris A, Gutierrez MC, et al. Gene expression patterns in formalin-fixed, paraffin-embedded core biopsies predict docetaxel chemosensitivity in breast cancer patients. Breast Cancer Res Treat 2008;108:233-40.

51. Soran A, Bhargava R, Johnson R, et al. The impact of Oncotype $\mathrm{DX}(\mathrm{R})$ recurrence score of paraffin-embedded core biopsy tissues in predicting response to neoadjuvant chemotherapy in women with breast cancer. Breast Dis 2016;36:65-71.

52. Straver ME, Glas AM, Hannemann J, et al. The 70gene signature as a response predictor for neoadjuvant chemotherapy in breast cancer. Breast Cancer Res Treat 2010;119:551-8.

53. Parker JS, Mullins M, Cheang MCU, et al. Supervised risk predictor of breast cancer based on intrinsic subtypes. J Clin Oncol 2009;27:1160-7.

54. Tsunashima R, Naoi Y, Kagara N, et al. Construction of multi-gene classifier for prediction of response to and prognosis after neoadjuvant chemotherapy for estrogen receptor positive breast cancers. Cancer lett 2015;365:166-73.

55. Bear HD, Wan W, Robidoux A, et al. Using the 21-gene assay from core needle biopsies to choose neoadjuvant therapy for breast cancer: A multicenter trial. J Surg Oncol 2017;115:917-23.

56. von Minckwitz G, Blohmer JU, Costa SD, et al. Responseguided neoadjuvant chemotherapy for breast cancer. J Clin Oncol 2013;31;3623-30.

57. Masuda N, Lee S-J, Ohtani S, et al. Adjuvant capecitabine for breast cancer after preoperative chemotherapy. N Engl J Med 2017;376:2147-59.

58. Toi M, Ishida T, Ito $\mathrm{Y}$, et al. Addition of S1 to endocrine therapy in the post-operative adjuvant treatment of hormone receptor-positive and human epidermal growth factor receptor 2-negative primary breast cancer: A multicenter, open-label, phase 3 randomized trial (POTENT trial). San Antonio Breast Cancer Symposium 2019. 